---مجلة علوم الرافدين، المجلد 27، العدد 4 / عدد خاص بالمؤتمر العلمي الثالث لعلوم الحياة، ص 76-87، 2018----

السلوك الوراثي والمظهري في الصفات الكمية لبعض أصناف من الحنطة (Triticum aestivum L.) وهجنها التبادلية النصفية

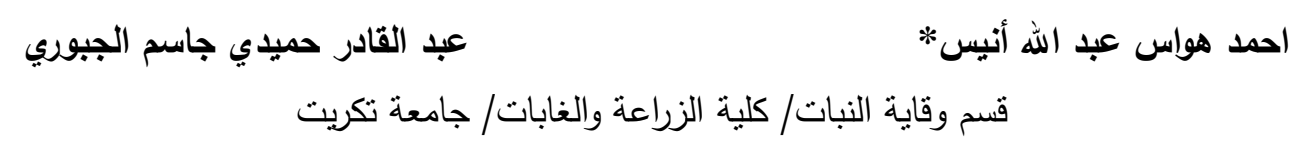

*E-mail: $\underline{\text { Ahmed75hawas@yahoo.com }}$

(أُنتلم 2018/6/6 ؛ قُبْل 2018/11/1)

نفذ البحث في حقول قسم المحاصيل الحقلية في كلية الزراعة في جامعة تكريت خلال الموسمين (2011-2012 و2012-2013) كان الأول لغرض إجراء التهجين التبادلي النصفي والآخر لتقييم الآباء مع هجنها باستخدام ستة نراكيب وراثية

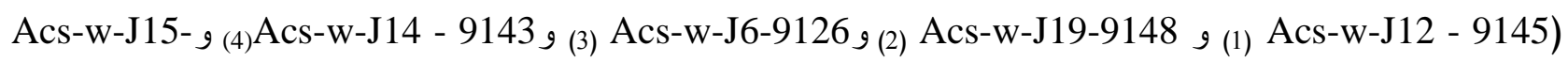
9144(5) وشام 6 (6)) بهدف دراسة السلوك الوراثي والمظهري لصفات عدد الأيام لطرد السنابل وارتفاع النبات وعدد سنابل النبات

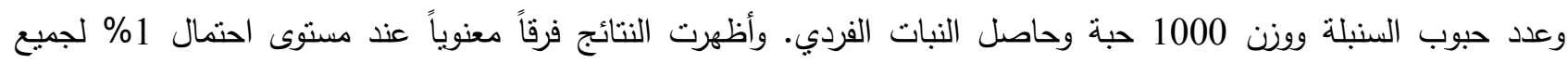
الصفات المدروسة، وتميزت الآباء بأعلى المنوسطات الحسابية وبتفوق معنوي إضافة الى تأثثرات المقدرة العامة في الاتحاد كالأب

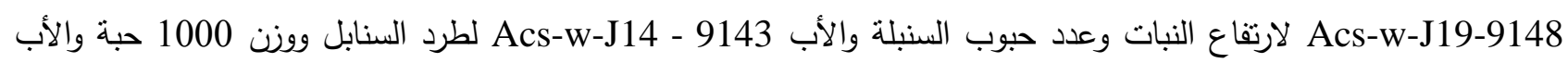
Acs-W-J15-9144 لعدد حبوب السنبلة وحاصل النبات الفردي، وكذلك تفوق الهجن معنوياً في أدائها وتأثيراتها بالاتجاه المرغوب وحصولها على قوة هجين ذو دلالة إحصائية ومرغوبة كالهجين (X Acs-w-J19-9148 Aام6) لعدد حبوب السنبلة وحاصل النبات الفردي والهجين (X Acs-w-J15-9144 شام6) لوزن 1000 حبة وحاصل النبات الفردي. وان جميع الصفات

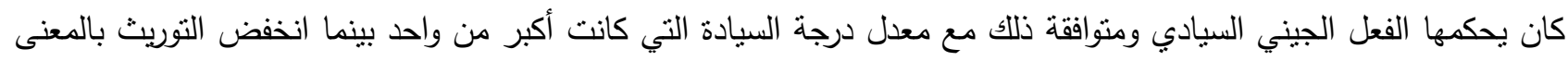

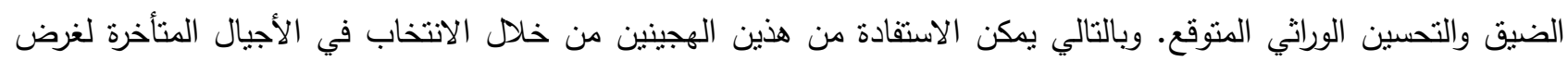
تطوير أصناف هجينة عالية الإنتاج مستقبلاً.

الكلمات الادالة: الحنطة، التهجين التبادلي، قوة الهجين، المعالم الوراثية.

\title{
Genotypic and Phenotypic Behavior for Quantitative Traits of some Wheat Varieties (Triticum aestivum L.) and their Half Diallel Crosses
}

\author{
Ahmed H. Anees \\ Abdul-Khader H. AL- Jubouri \\ Department of Plant Protection/College of Agriculture and Forestry/ University of Tikrit
}

\begin{abstract}
A field experiment was carried out at the research station of the field crops department $\backslash$ college of agricultureluniversity of Tikrit during (2011-2012 and 2013 winter seasons, included crossing according half diallel cross in the first season and evaluating parents and their crosses in the second season to study the genotypic and phenotypic behavior for the traits: days to anthesis, plant height $(\mathrm{cm})$, no. spikes. plant ${ }^{-1}$, no. grains. spike ${ }^{-1}, 1000$ grains weight $(\mathrm{g})$ and individual grain
\end{abstract}




$$
\text { احمد هواس عبد الله أنيس و عبد القادر حميدي جاسم الجبوري }
$$

weight. Results showed highly significant differences for whole studied traits, many parents have high significant means and agood combiner in many traits especially ACS-W-J19-9148 parents in plant height and grains. spike ${ }^{-1}$, ACS-W-J14-9143 parent in days to anthesis and 1000 grain weight and ACS-W-J15-9144 in no.grains.spike ${ }^{-1}$ and individual grain weight. Many crosses have better performance and affected positively to wards desired direction of significant hybrid vigor (ACS-WJ19-9148 X sham 6) in no. grains. spike ${ }^{-1}$ and individual grain yield, (ACS-W-J15-9144) in 1000 grain weight and individual grain weight. Whole traits were controlled by dominance type of gene action and that adequate with the ratio of the degree of dominance which were more than one. Consequently heritability in narrow sense and expected genetic advance were low. The last two crosses can be useful through the application of pedigree selection on the late segregation generations to improve bread wheat cultivars.

Keywords: Wheat, half diallel crossing, hybrid vigor, genetic parameters.

\section{المقدمة}

تعد الحنطة Triticum aestivum Lن أهم محاصيل الحبوب، وعلى الرغم من الزيادة الكبيرة في إنتاجها إلا أن ذلك لا

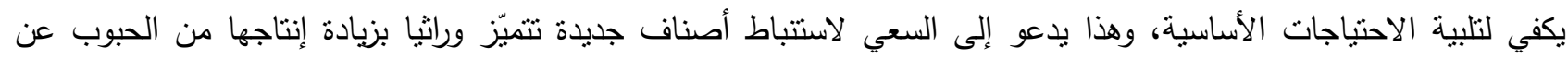

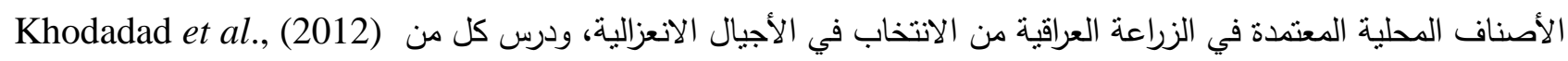
والعطرات وآخرون (2014) و (2015) Kalhro et al., وأنيس وآخرون (2017) حول أداء الأصناف في هجنها التبادلية

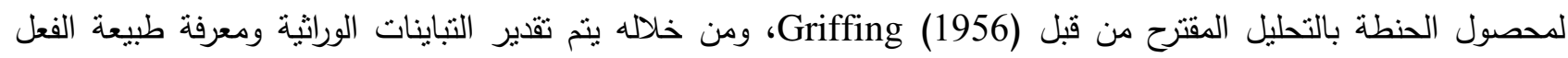

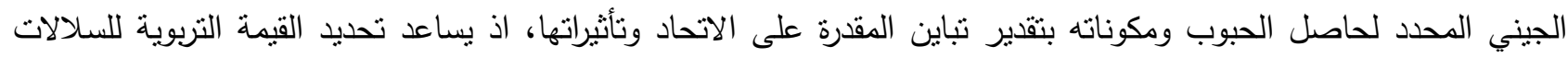

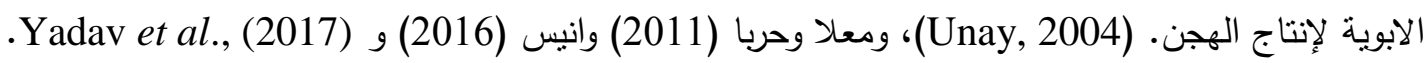
وتعد معرفة الأهمية النسبية للفعلين الإضافي والسيادي ودرجة التوريث ومعدل درجة السيادة والتحسين الوراثي المتوقع الخطوة الأهم من خلا التخطيط لبرامج التحسين الوراثي، والذي يمكن المربي من التتبؤ بمقدار التحسين الوراثي الناتج من عملية الانتخاب (Yaday and Singh, 2011)، واستعمل هذه المعالم الوراثية في محصول الحنطة باحثون عدة ومنهم البدراني وآخرون

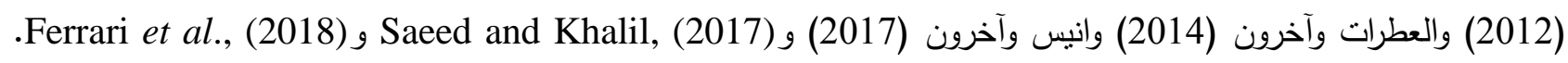

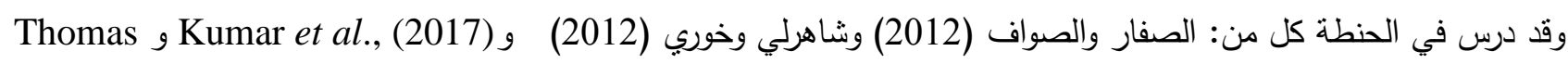
.et al., (2017)

ويهدف البحث إلى معرفة أداء الآباء وهجنها التبادلية النصفية بتقدير درجة التوريث الحاصل ومكوناته وتقدير المقدرة العامة والخاصة وتأثيراتها وقوة الهجين.

\section{المواد وطرائق العمل}

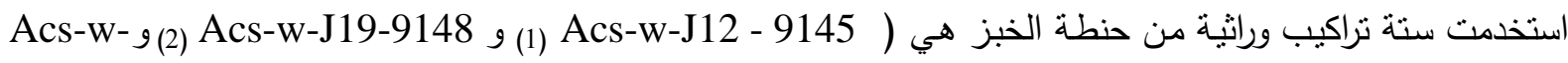

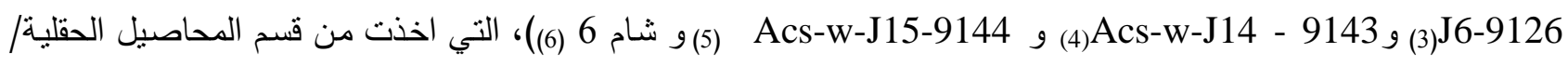

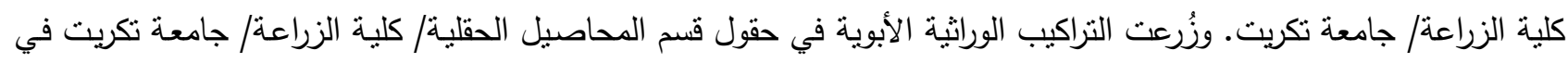
منتصف تشرين الثاني للموسم 2011-2012، وعند موسم التزهير أُجريت جميع التهجينات الممكنة بينها دون الهجن العكسية وفقاً لطريقة Griffing الثانية، وعند النضـج حصدت النباتات وتم الحصول على بذور الجيل الأول لتلك التهجينات النصفية البالغة خمسة عشر هجيناً وتم تفريط الحبوب من السنابل والاحتفاظ بها للزراعة في الموسم القادم. وزرعت بذور الأصناف الأبوية الستة

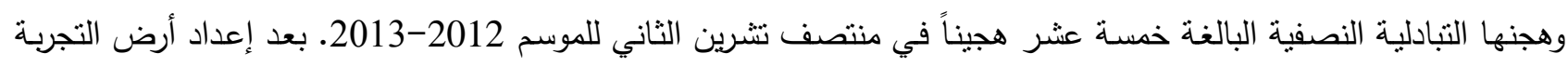

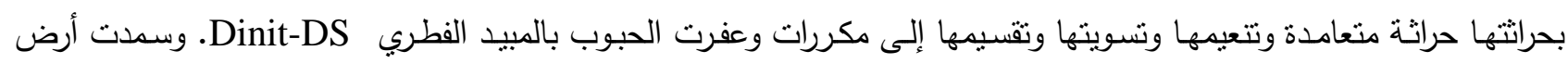




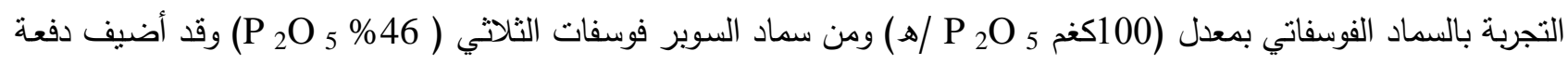

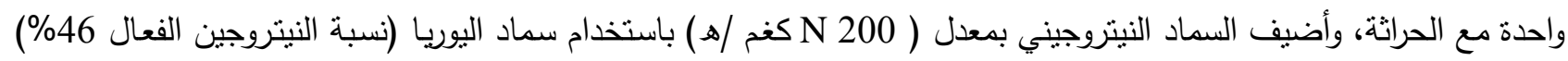
بدفعتين (سباهي، Randomized Complete Block Design بثناثنة مكررات، ضم كل مكرر واحداً وعشرين خطاً طول كل منها مترين (فكل خط لنمط وراثي واحد) وزرع في كل خط عشرين نباتاً بمسافة 10سم بين نبات وآخر ، و 60 سم بين خط وآخر • ووزعت الأنماط الوراثية على الخطوط بطريقة عشوائية، وسيج الحقل وغطي بشبكة لحمايته من أضرار الطيور والحيوانات. وأجريت جميع العمليات الضرورية من خدمة التربة والمحصول جميعه خلال

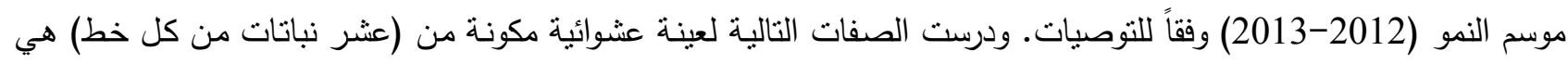
المدة لطرد السنابل (وسُجلَ وقت طرد السنابل بالأيام اعتباراً من أول يوم لري النبات وحتى اجتياز السنبلة الأولى قاعدة ورقة فئة

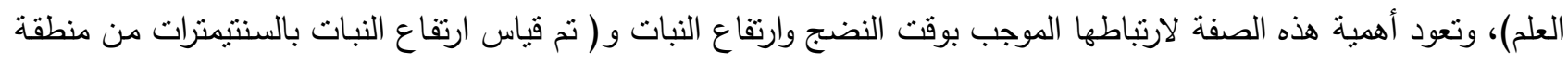
تاج الجذور إلى قمة أعلى سنبلة في النبات دون السفا) وعدد السنابل في النبات و (تم عد السنابل المحمولة على الأشطاء التي زاد التهات

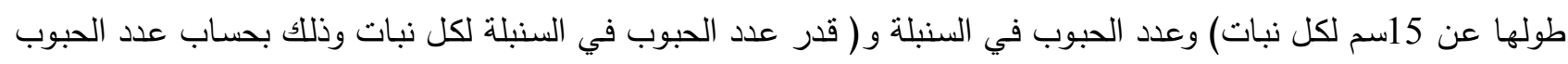

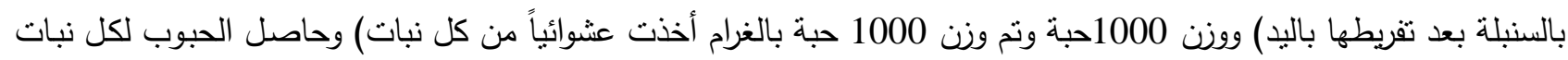
ثم و (عين حاصل الحبوب بالغرام من الوزن الجاف للحبوب الناتجة من كل نبات).

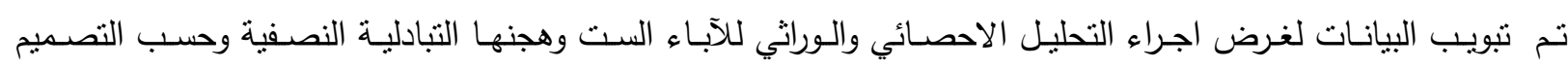

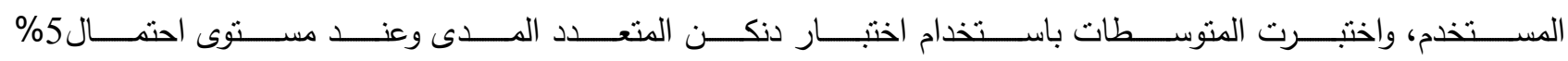

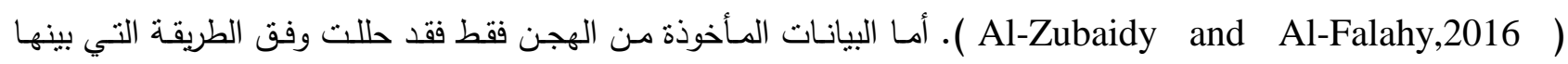

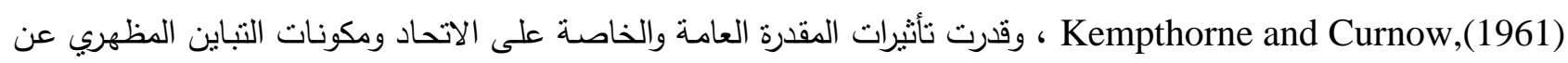

$\operatorname{MSGCA}=\sigma^{2} \mathrm{e}+\mathrm{r} \sigma^{2} \mathrm{~s}+[\mathrm{rs}(\mathrm{n}-2) / \mathrm{n}-1] \sigma^{2} \mathrm{~g}$ طريق متوسط التباين المتوقع وحسب المعادلات الآثية:

MSSCA $=\sigma^{2} \mathrm{e}+\mathrm{r} \sigma^{2} \mathrm{~s}$

$\mathrm{MSe}=\sigma^{2} \mathrm{e}$

حيث أن: MSGCA و MSSCA و هي متوسط المربعات للمقدرة العامة والخاصة على الاتحاد والخطأ التجريبي على ألى الترتيب، بعد إيجاد قيم $\sigma^{2} \mathrm{E}=\mathrm{MSe} \quad ; \quad \sigma^{2} \mathrm{~A}=2 \sigma^{2} \mathrm{~g} \quad ; \quad \sigma^{2} \mathrm{D}=\sigma^{2} \mathrm{~s}$

واختبرت معنوية هذه المكونات عن الصفر وفق طريقة (Kempthorne, (1957) وقدرت نسبة التوريث بمعناها الضيق والواسع BSh ${ }^{2}$ ومعدل درجة السيادة للصفات المختلفة بنطبيق المعادلات الآتية:

$$
h^{2} \mathrm{NS}=\sigma^{2} \mathrm{~A} / \sigma^{2} \mathrm{P} \quad ; \quad h^{2} \mathrm{BS}=\sigma^{2} \mathrm{G} / \sigma^{2} \mathrm{P} \quad ; \quad \overline{\mathrm{a}}=\sqrt{ } 2 \sigma^{2} \overline{\mathrm{D} / \sigma^{2} \mathrm{~A}}
$$$$
\sigma^{2} \mathrm{G}=\sigma^{2} \mathrm{~A}+\sigma^{2} \mathrm{D}
$$$$
\sigma^{2} \mathrm{P}=\sigma^{2} \mathrm{G}+\sigma^{2} \mathrm{E}
$$

$$
\text { علماً ان: G }
$$

وتم اعتماد حدود التوريث بالمعنى الضيق حسب العذاري (1999) (أقل من 20\% منخفض ومن 20\%-50\% منوسط

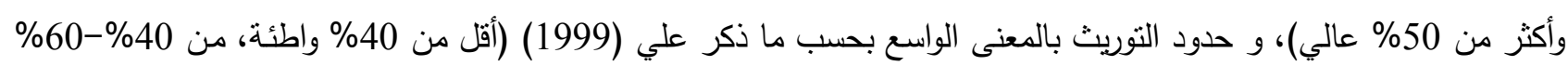

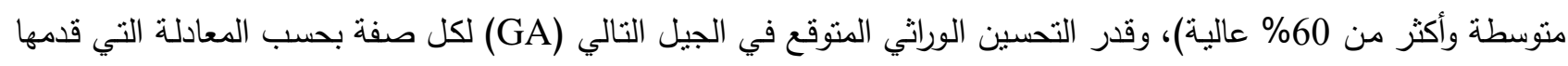

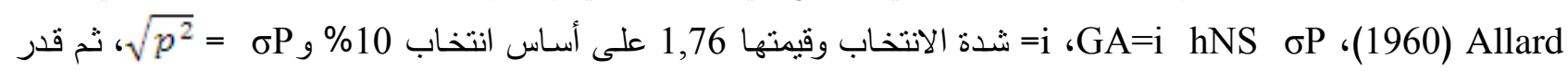

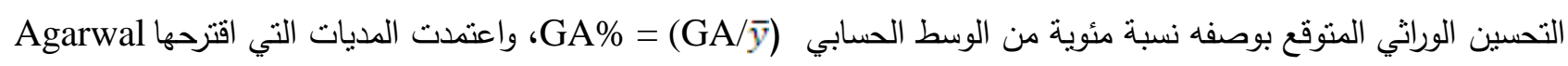

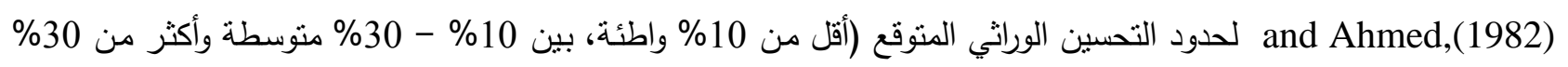




$$
\text { احمد هواس عبد الله أنيس و عبد القادر حميدي جاسم الجبوري }
$$

عالية)، اما قوة الهجين فقدرت على أساس المقارنة مع أفضل اب واختبرت معنويتها باستخدام اختبار t ـ تمت الاستعانة في انجاز التحاليل الإحصائية والوراثية بالبرامج الإحصائية الجاهزة (SAS version 9)

.Microsoft Office Excel 2003 ,

\section{النتائج والمناقشة}

يتضح من (الجدول 1) تحليل التباين للآباء وهجنها التبادلية النصفية للصفات المدروسة، ونلاحظ ان مصدري الآباء والهجن كان معنوياً عند مستوى احتمال 1\% لجميع الصفات المدروسة، وان هذا التغاير كان بسبب اختلافها للمورثات التي تمنلكها لهذه الصفات وكذلك هذه الاختلافات كانت بسبب التباعد الوراثي بين الإباء وانعكس ذلك عن اختلاف الهجن الناتجة في استجابتها للصفات الكمية (الجدول 8)، مما يستوجب الاستمرار في دراسة السلوك الوراثي لها، وهذه النتائج تتوافق مع ما ذكره كل

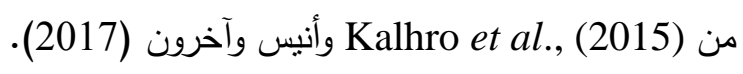

الجدول 1: تحليل التباين للآباء والهجن ممثلاً بمتوسط المربعات (MS) للصفات قيد الدراسة

\begin{tabular}{|c|c|c|c|c|c|c|c|}
\hline الفردي النبات & حزن 1000 & عدلد حبوب & علند سنابل & ارتفاع النبات & السنابل الأيام لطرد & /لصفات / & مصادر \\
\hline 8.35 & 9.41 & 6.41 & 0.60 & 415.27 & 5.38 & 2 & المكررات \\
\hline$* * 24.47$ & $* * 19.92$ & $* * 48.66$ & $* * 21.62$ & $* * 229.18$ & $* * 18.48$ & 5 & الإباء \\
\hline 1.11 & 1.79 & 3.69 & 0.04 & 18.48 & 0.52 & 10 & الخطأ التجريبي \\
\hline 26.17 & 10.08 & 19.16 & 1.32 & 57.73 & 19.28 & 2 & المكررات \\
\hline$* * 35.22$ & $* * 33.82$ & **91.70 & $* * 1.17$ & $* * 155.48$ & $* * 21.49$ & 15 & الهجن \\
\hline 0.87 & 0.42 & 0.70 & 0.16 & 2.70 & 0.52 & 30 & الخطأ التجريبي \\
\hline
\end{tabular}

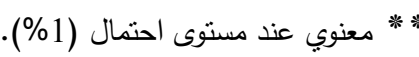

ونلحظ من (الجدول 2) إن الأبوين 4 و 6 أبكر الأصناف باختلاف معنوي عن بقية الآباء بمتوسط قدره 117.00 يوم

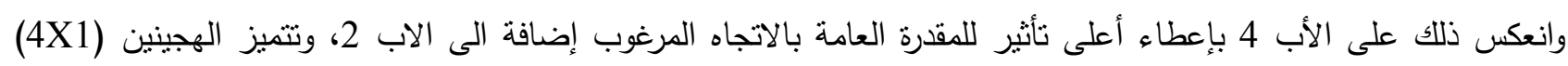
و (4X2) وبلغا 116.66 يوم لكليهما، ونأثثرات المقدرة الخاصة في الاتحاد كانت مختلفة عن الصفر وبالاتجاه المرغوب للهجائن

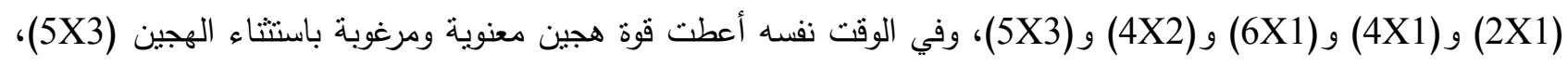

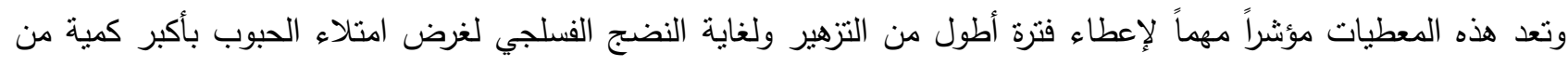

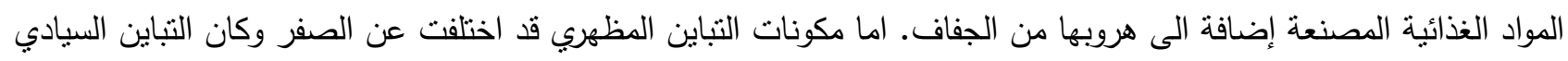
اعلى من التباين الإضافي وهذا ما لوحظ من خلال معلمي التوريث بالمعنى الضيق الذي كان متوسطاً، ومعدل درجة السيادة الذي الذي كان اكبر من واحد مع انخفاض قيمة التحسين الوراثي المتوقع، ويعد التحسين الوراثي لهذه الصفة استراتيجية تربوية لتعزيز ثباتية

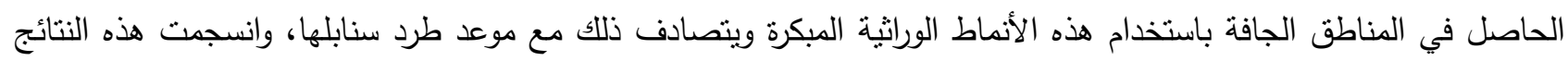

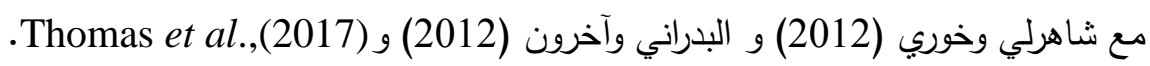


الجدول 2: متوسطات الإباء وهنها وتأثيراتهما وقوة الهجين لصفة عدد الأيام لطرد السنابل (يوم)

\begin{tabular}{|c|c|c|c|c|c|c|c|c|}
\hline \multicolumn{2}{|c|}{ مكونات التباين المظهري } & \multirow{2}{*}{ قالمقارنة مع الهين } & \multirow{2}{*}{$\begin{array}{r}\text { تأثير المقدرة } \\
\text { الخاصة } \\
3.54-\end{array}$} & \multirow{2}{*}{ 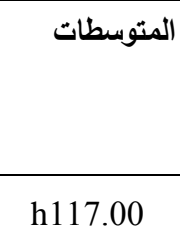 } & \multirow{2}{*}{ الهجن } & \multirow{2}{*}{$\begin{array}{c}\text { تأثثير المقرة } \\
\\
\end{array}$} & \multirow{2}{*}{ المتوسطات } & \multirow{2}{*}{$\begin{array}{l}\text { الإباء } \\
1\end{array}$} \\
\hline 0.18 & $\sigma_{E}^{2}$ & & & & & & & \\
\hline 0.11 & S.E & $* * 1.89$ & 2.66 & a125.33 & $3 \times 1$ & & & \\
\hline 2.75 & $\sigma_{A}^{2}$ & $* * 5.14$ & $2.58^{-}$ & h116.66 & $4 \times 1$ & & & \\
\hline 0.78 & S.E & $* 1.08$ & 2.87 & ab124.33 & $5 \times 1$ & & & \\
\hline 5.18 & $\sigma_{D}^{2}$ & $* * 3.52-$ & $1.91-$ & g118.66 & $6 \times 1$ & & & \\
\hline 1.84 & S.E & 0.82 & 0.87 & c122.33 & $3 \times 2$ & \multirow{4}{*}{$0.50^{-}$} & \multirow[t]{4}{*}{ b120.66 } & \multirow[t]{4}{*}{2} \\
\hline \multirow[b]{2}{*}{7.93} & $\sigma_{G}^{2}$ & $* * 3.31-$ & $1.37-$ & h116.66 & $4 \times 2$ & & & \\
\hline & & $0.27-$ & 0.08 & ef120.33 & $5 \times 2$ & & & \\
\hline \multirow[t]{2}{*}{8.11} & $\sigma_{0}^{2}$ & 0.00 & 1.29 & ef 120.66 & $6 \times 2$ & & & \\
\hline & & $* 1.09$ & $0.16^{-}$ & ef 120.00 & $4 \times 3$ & \multirow[t]{3}{*}{1.62} & \multirow[t]{3}{*}{ b121.33 } & \multirow[t]{3}{*}{3} \\
\hline \multirow[t]{2}{*}{0.98} & $h_{B S}^{2}$ & 0.00 & $1.04-$ & cde121.33 & $5 \times 3$ & & & \\
\hline & & $* * 1.92$ & 2.16 & b123.66 & $6 \times 3$ & & & \\
\hline 0.34 & $h_{N g}^{2}$ & $* * 2.25$ & 2.04 & $\operatorname{def} 121.00$ & $5 \times 4$ & $1.79-$ & $\mathrm{c} 117.00$ & 4 \\
\hline 1.94 & $\bar{a}$ & $* * 2.27$ & 1.58 & fg119.66 & $6 \times 4$ & 0.41 & c118.33 & 5 \\
\hline 1.70 & $\overline{G_{\tilde{i}}}$ & $* * 3.09$ & 1.70 & cd122.00 & $6 \times 5$ & $0.04-$ & $\mathrm{c} 117.00$ & 6 \\
\hline 1.41 & $G_{i} \%$ & 4.87- & 0.17 & S.E & & 0.13 & S.E & \\
\hline
\end{tabular}

يبين(الجدول 3) صفة ارتفاع النبات حيث تفوق الاب 4 معنويا (106.00 سم) على الاب 6، وعلى العكس من ذلك ابدى الابوان 2 و 3 تأثيراتهما في الاتجاه المرغوب، وليس بالضرورة ان الإبـاء التي تعطي اعلى المتوسطات تكون ذو اتحاد التهاد معنويا

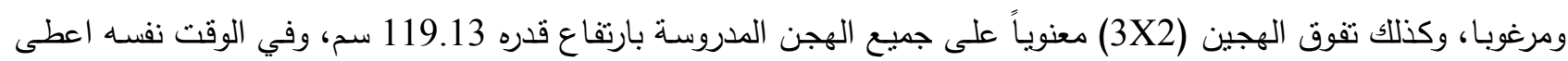

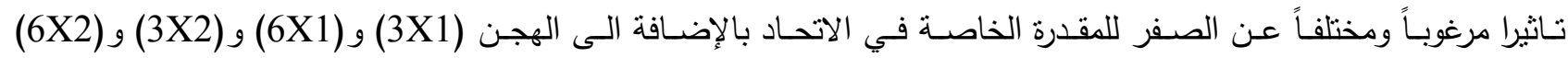

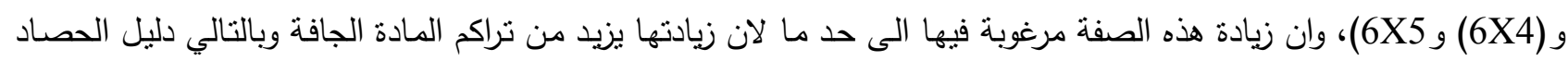
Harvest index النباتات التي تتعرض للمنافسة يقل فيها ارتفاع النبات، وبالتالي عوامل النمو التي تصب في زيادة الجزء الاقتصادي مما بعطي لهني

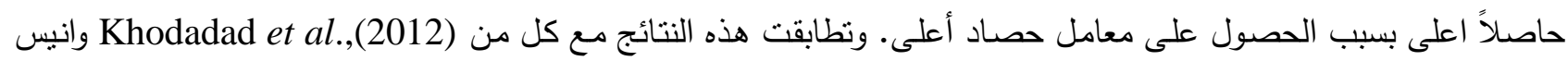
(2016) و Yadav et al., (2017) بعكس المعطيات التي ذكرت فلم تحقق أي من الهجن قوة هجين معنوية بالاتجاه المرغوب. ونلحظ ان التباينات البيئية والإضافية والسيادية اختلفت عن الصفر وكان التباين الإضافي والسيادي مثقارباً ويدل ذلك على ان ان هذه الهن

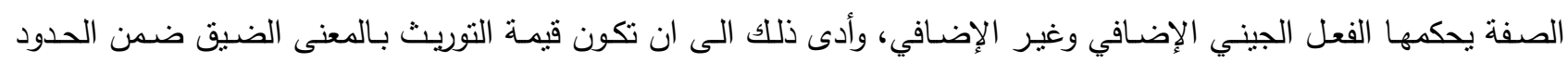
المتوسطة وان معدل درجة السيادة كانت قريبة من واحد، وبالتالي يكون تحسين هذه الصفة من خلال الانتخاب كائنا في الأجيال 
احمد هواس عبد الله أنبس و عبد القادر حميدي جاسم الجبوري

الجدول 3: متوسطات الآباء وهجنها وتأثيراتهما وقوة الهجين لصفة ارتفاع النبات (سم)

\begin{tabular}{|c|c|c|c|c|c|c|c|c|}
\hline \multicolumn{2}{|c|}{ مكونات التباين المظهري } & \multirow{2}{*}{ قالمقارنة الهجين } & \multirow{2}{*}{ تأثير المقدرة } & \multirow{2}{*}{ المتوسطات } & \multirow{2}{*}{\begin{tabular}{|l} 
الهجن \\
2 X 1
\end{tabular}} & \multirow{2}{*}{ تأثير المقدرة } & \multirow{2}{*}{ المتوسطات } & \multirow{2}{*}{$\begin{array}{c}\text { الإباء } \\
\\
1\end{array}$} \\
\hline 2279 & $\sigma^{2}$ & & & & & & & \\
\hline 14.92 & S.E & 11.42 & 9.66 & b116.10 & $3 \times 1$ & & & \\
\hline 31.06 & $\sigma_{A}^{2}$ & $11.13-$ & $7.61-$ & i94.20 & $4 \times 1$ & & & \\
\hline 10.08 & S.E & $6.38-$ & $3.94-$ & gh98.16 & $5 \times 1$ & & & \\
\hline 30.23 & $\sigma_{D}^{2}$ & 11.49 & 13.14 & cd106.66 & $6 \times 1$ & & & \\
\hline 18.86 & S.E & 14.33 & 10.92 & a119.13 & $3 \times 2$ & 1.88 & ab99.33 & 2 \\
\hline 61.29 & $\sigma_{G}^{2}$ & 6.79 & $4.78^{-}$ & gh98.80 & $4 \times 2$ & & & \\
\hline & & $0.92-$ & 0.02 & de103.90 & $5 \times 2$ & & & \\
\hline 84.08 & $\sigma_{0}^{2}$ & 0.80 & 4.83 & fg100.13 & $6 \times 2$ & & & \\
\hline & & 1.13 & 0.53 & c107.20 & $4 \times 3$ & 4.96 & ab104.20 & 3 \\
\hline & $h_{B S}^{2}$ & 2.35 & $4.56^{-}$ & ef 102.40 & $5 \times 3$ & & & \\
\hline 0.73 & & $7.86-$ & $2.37-$ & hi96.00 & $6 \times 3$ & & & \\
\hline 0.37 & $h_{\text {Ns }}^{2}$ & -2.138 & 1.39 & de103.73 & $5 \times 4$ & 0.34 & a106.00 & 4 \\
\hline 1.40 & $\bar{a}$ & $9.11-$ & 2.57 & hi96.33 & $6 \times 4$ & 0.63 & ab104.87 & 5 \\
\hline 5.96 & $G_{i}$ & $7.81-$ & 2.61 & hi96.66 & $6 \times 5$ & $7.94-$ & $\mathrm{b} 75.07$ & 6 \\
\hline 5.88 & $G_{i} \%$ & & 2.01 & S.E & & 1.54 & S.E & \\
\hline
\end{tabular}

وتقوق الابوان 1 و 5 معنوياً (10.19 سنبلة) على الابوين (2و6) ولم يختلفا عن الابوين (3و4) في صفة عدد سنابل النبات (الجدول 4)، وتأثيرات المقدرة العامة في الاتحاد كانت مرغوبة ومختلفة عن الصفر لثناثة اباء (1 و 2 و4)، وان جميع الانيع

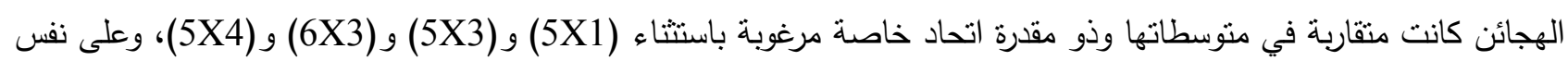

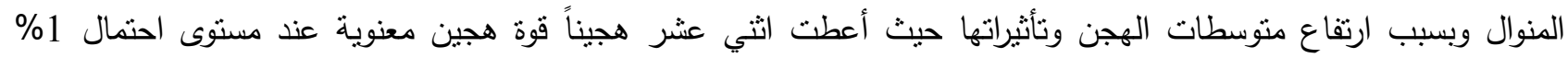
وبالاتجاه المرغوب ماعدا (5X1) و (5X3) و (5X4)، وهذا يرجع الى وجود تباعد وراتي عالٍ بين التراكيب الابوية، وقد نم تأكيد

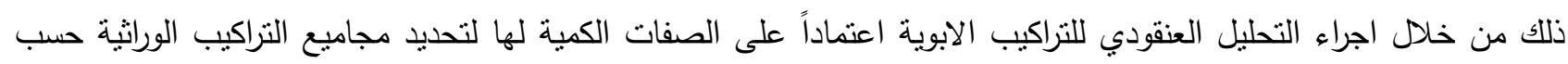

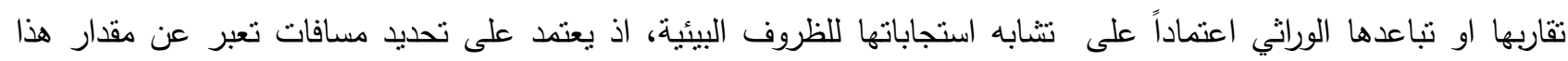
التباعد حسب أدائها المظهري واصولها الوراثية، وبناء على قيم البعد الوراثي تم ايجاد العلاقة الوراثية التي تربطها على بشكل

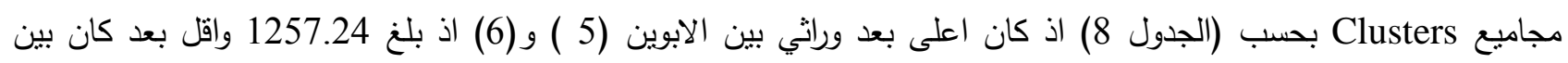

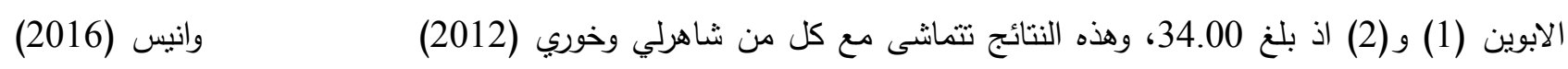

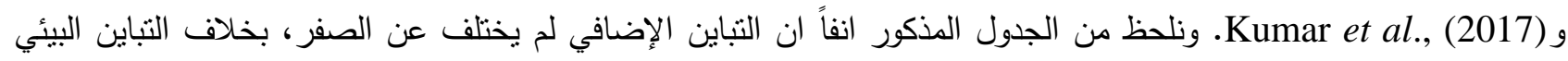
والسيادي، وكان الأخير أعلى من التباين الإضافي وتتبين أهمية تأثثر الجانب الوراثي في الجزء السيادي للجينات، لمعرفة الإني أيهما

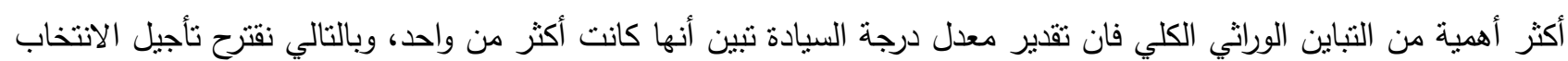
لهذا الصفة إلى الأجيال المتأخرة. 
الجدول 4: متوسطات الآباء وهجنها وتأثيراتهما وقوة الهجين لصفة عدد سنابل النبات

\begin{tabular}{|c|c|c|c|c|c|c|c|c|}
\hline \multicolumn{2}{|c|}{ مكونات التباين المظهري } & \multirow{2}{*}{ قالمقارنة مع الهين } & \multirow{2}{*}{ تأثير المقدرة } & \multirow{2}{*}{$\begin{array}{l}\text { المتوسطات } \\
\text { a12.56 }\end{array}$} & \multirow{2}{*}{$\begin{array}{l}\text { الهجن } \\
\\
2 \text { X } 1\end{array}$} & \multirow{2}{*}{$\begin{array}{r}\text { تأثير المقدرة } \\
\\
\\
\\
\end{array}$} & \multirow{2}{*}{$\begin{array}{l}\text { المتوسطات } \\
\text { a10.19 }\end{array}$} & \multirow{2}{*}{$\begin{array}{l}\text { الإباء } \\
1\end{array}$} \\
\hline 0.05 & $\sigma_{E}^{2}$ & & & & & & & \\
\hline 0.03 & S.E & $* * 24.13$ & 1.02 & a12.65 & $3 \times 1$ & & & \\
\hline 0.04 & $\sigma_{A}^{2}$ & $* * 24.26$ & 0.90 & a12.66 & $4 \times 1$ & & & \\
\hline 0.09 & S.E & 9.25 & $0.38^{-}$ & b11.13 & $5 \times 1$ & & & \\
\hline 1.92 & $\sigma_{D}^{2}$ & $* * 24.13$ & 1.13 & a12.65 & $6 \times 1$ & & & \\
\hline 0.68 & S.E & $* * 22.07$ & 0.70 & a12.22 & $3 \times 2$ & 0.10 & b9.60 & 2 \\
\hline 1.96 & $\sigma^{2}$ & $* * 22.97$ & 0.57 & a12.22 & $4 \times 2$ & & & \\
\hline & & $* * 21.58$ & 0.98 & a12.39 & $5 \times 2$ & & & \\
\hline 2.01 & $\sigma^{2}$ & $* * 27.58$ & 0.95 & a12.35 & $6 \times 2$ & & & \\
\hline & & $* * 25.10$ & 1.04 & a12.52 & $4 \times 3$ & $006-$ & ab10.01 & 3 \\
\hline 0.98 & $h_{B S}^{2}$ & 8.82 & $0.14^{-}$ & b11.09 & $5 \times 3$ & & & \\
\hline & & $* 12.55$ & 0.03 & b11.26 & $6 \times 3$ & & & \\
\hline 0.02 & $h_{N s}^{2}$ & 9.68 & $0.19-$ & b11.18 & $5 \times 4$ & 0.07 & ab9.93 & 4 \\
\hline 9.78 & $\bar{a}$ & $* * 24.72$ & 1.02 & a12.39 & $6 \times 4$ & $0.16^{-}$ & a10.19 & 5 \\
\hline 0.05 & $G_{\tilde{i}}$ & $* * 24.98$ & 1.61 & a12.74 & $6 \times 5$ & $0.17-$ & $\mathrm{c} 8.73$ & 6 \\
\hline 0.44 & $G_{i} \%$ & & 0.09 & S.E & & 0.06 & S.E & \\
\hline
\end{tabular}

يوضح (الجدول 5) تميز الإباء 1 و 2 و 5 بأعلى منوسط حسابي اذ بلغت (51.75 و53.09 و53.48 حبة/ سنبلة) وباختلاف معنوي عن بقية الإباء، وأبدى الأبوين 2 و5 بالإضافة الى الاب 6 نأنثاءثيرات للمقدرة العامة في الاتحاد، وتفوق الهجين (2X1)

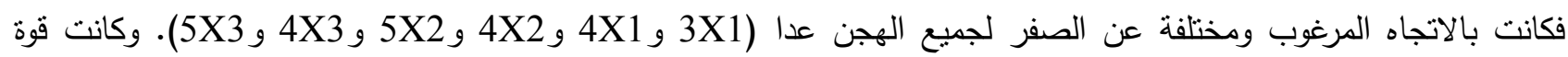

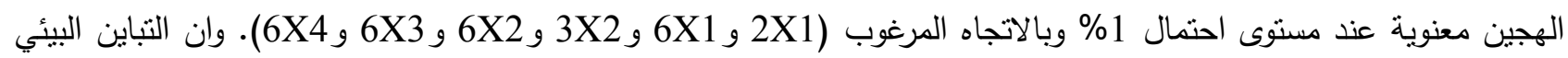

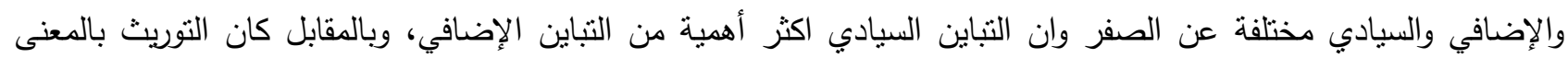

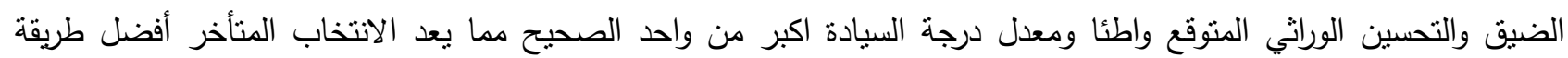
لزيادة تكرار الجينات المرغوبة في تحسين هذه الصفة، ويتوافق ذلك مع ما نوصل اليه انيس وآخرون (2017) و Saeed and .Ferrari et al., (2018) Khalil, (2017) 
احمد هواس عبد الله أنبس و عبد القادر حميدي جاسم الجبوري

الجدول 5: متوسطات الآباء وهجنها وتأثيراتهما وقوة الهجين لصفة عدد حبوب السنبلة

\begin{tabular}{|c|c|c|c|c|c|c|c|c|}
\hline \multicolumn{2}{|c|}{ مكونات التباين المظهري } & \multirow{2}{*}{ 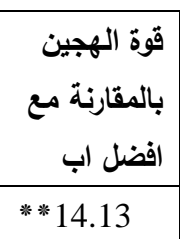 } & \multirow{2}{*}{ تأثير المقدرة } & \multirow{2}{*}{$\begin{array}{l}\text { المتوسطات } \\
\text { a60.60 } \\
\end{array}$} & \multirow{2}{*}{$\begin{array}{l}\text { الهجن } \\
2 \text { X } 1\end{array}$} & \multirow{2}{*}{ 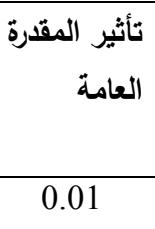 } & \multirow{2}{*}{$\begin{array}{l}\text { المتوسطات } \\
\text { a51.75 } \\
\end{array}$} & \multirow{2}{*}{$\begin{array}{l}\text { الإباءء } \\
1\end{array}$} \\
\hline 0.50 & $\sigma_{E}^{2}$ & & & & & & & \\
\hline 0.33 & S.E & $* * 10.60$ & $4.21^{-}$ & g46.26 & $3 \times 1$ & & & \\
\hline 6.46 & $\sigma_{A}^{2}$ & $* * 18.07-$ & 7.32- & i42.39 & $4 \times 1$ & & & \\
\hline 2.15 & S.E & $0.67-$ & 0.40 & $\mathrm{e} 53.12$ & $5 \times 1$ & & & \\
\hline 28.36 & $\sigma_{D}^{2}$ & $* * 13.95$ & 6.07 & b58.97 & $6 \times 1$ & & & \\
\hline 9.90 & S.E & $* * 11.23$ & 5.93 & b59.05 & $3 \times 2$ & 2.65 & a53.09 & 2 \\
\hline 3483 & $\sigma_{G}^{2}$ & $2.58-$ & $0.63-$ & ef51.72 & $4 \times 2$ & & & \\
\hline 34.83 & & $3.6-1$ & $3.80^{-}$ & ef51.55 & $5 \times 2$ & & & \\
\hline 35.32 & $\sigma_{p}^{2}$ & *7.07 & 1.30 & c56.85 & $6 \times 2$ & & & \\
\hline & & $* 7.66$ & $3.59-$ & $\mathrm{h} 44.47$ & $4 \times 3$ & $1.64-$ & $\mathrm{b} 47.73$ & 3 \\
\hline 0.99 & $h_{B S}^{2}$ & $4.50-$ & 0.01 & $\mathrm{f} 51.08$ & $5 \times 3$ & & & \\
\hline & & ***15.80 & 4.03 & $\mathrm{~d} 55.28$ & $6 \times 3$ & & & \\
\hline 0.18 & $h_{N s}^{2}$ & $3.21-$ & 1.462 & ef51.76 & $5 \times 4$ & $2.40^{-}$ & b48.16 & 4 \\
\hline 2.96 & $\bar{a}$ & $* * 22.22$ & 8.37 & b58.86 & $6 \times 4$ & 0.59 & a53.48 & 5 \\
\hline 1.91 & $G_{i}$ & 2.92 & 1.56 & d55.05 & $6 \times 5$ & 0.78 & c42.99 & 6 \\
\hline 3.67 & $G_{i} \%$ & & 0.29 & S.E & & 0.22 & S.E & \\
\hline
\end{tabular}

ويشير (الجدول 6) الى ان الاب (4) تفوق معنوياً (41.36غر) على بقية الإباء وابدى تأثيراته للمقدرة العامة في الاتحاد

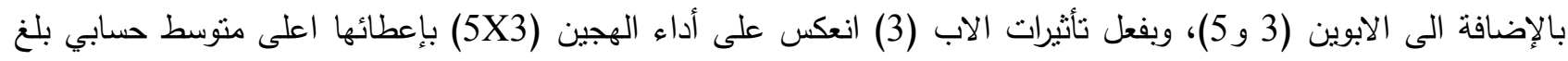

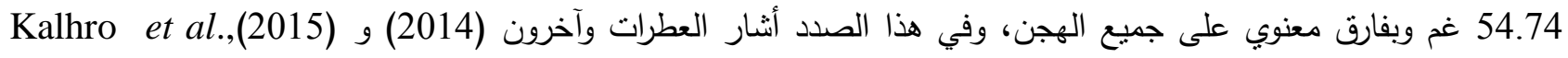
على أداء متفوق لبعض الإباء والهجن في هذه الصفة، وكذلك ظهور تأثثر للمقدرة الخاصة في الاتحاد كانت بالاتجاه المرغوب التهاب ومختلفة عن الصفر وتراوحت (7.43 - 0.26) باستثناء هجين واحد (6X3) كان سالباً، كما حققت جميع الهجائن قوة هجين

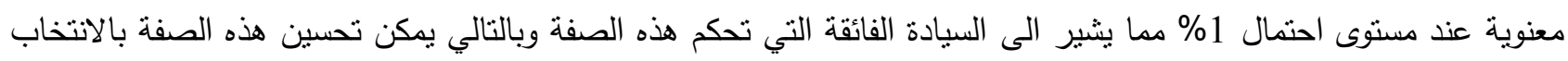

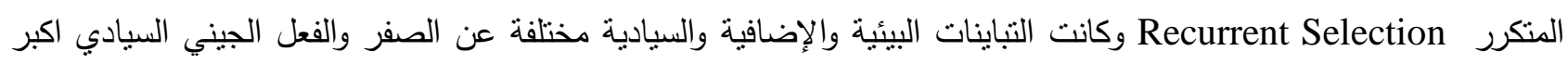
من الفعل الجيني الإضافي مما أدى الى انخفاض قيمة التوريث بالمعنى الضيق وارتفاع قيمة معدل درجة السيادة (اكبر من واحد) وكانت قيم التقام الوراثي ونسبته منخفضة، وعليه يمكن إجراء الانتخاب في الأجيال المتأخرة لتحسين هذه الصفة، وتتسجم هذه النتيجة مع البدراني وآخرون (2012). 
الجدول 6: متوسطات الآباء وهجنها وتأثيراتهما وقوة الهجين لصفة وزن 1000 حبة (غم)

\begin{tabular}{|c|c|c|c|c|c|c|c|c|}
\hline \multicolumn{2}{|c|}{ المظهوينات التباين } & قالمقارنة مع الهين & تأثير المقدرة & المتوسطات & الهجن & تأثير المقدرة & المتوسطات & الإباء \\
\hline 0.27 & $\sigma_{E}^{2}$ & $* * 21.93$ & 0.68 & $\mathrm{~g} 44.10$ & $2 \times 1$ & \multirow[t]{5}{*}{$1.86^{-}$} & \multirow[t]{5}{*}{$\mathrm{d} 33.87$} & \multirow[t]{5}{*}{1} \\
\hline 0.17 & S.E & $* * 33.39$ & 6.75 & $\mathrm{c} 51.50$ & $3 \times 1$ & & & \\
\hline 2.73 & $\sigma_{A}^{2}$ & $* * 13.45$ & 2.22 & $\mathrm{f} 46.92$ & $4 \times 1$ & & & \\
\hline 2.26 & S.E & $* * 38.47$ & 4.96 & de49.72 & $5 \times 1$ & & & \\
\hline 49.43 & $\sigma_{D}^{2}$ & $* * 21.14$ & 1.42 & g44.44 & $6 \times 1$ & & & \\
\hline 17.04 & S.E & **39.49 & 4.17 & cd50.46 & $3 \times 2$ & \multirow[t]{4}{*}{$0.33^{-}$} & \multirow{4}{*}{ bcd36.17 } & \multirow[t]{4}{*}{2} \\
\hline \multirow{2}{*}{52.16} & \multirow[t]{2}{*}{$\sigma_{G}^{2}$} & ***24.38 & 5.22 & c51.44 & $4 \times 2$ & & & \\
\hline & & $* * 29.35$ & 0.50 & f46.79 & $5 \times 2$ & & & \\
\hline \multirow[b]{2}{*}{52.43} & \multirow[t]{2}{*}{$\sigma_{p}^{2}$} & $* * 42.51$ & 6.99 & $\mathrm{c} 51.55$ & $6 \times 2$ & & & \\
\hline & & ***17.75 & 1.14 & $\mathrm{e} 48.70$ & $4 \times 3$ & \multirow[t]{3}{*}{0.99} & \multirow[t]{3}{*}{ b38.16 } & \multirow[t]{3}{*}{3} \\
\hline \multirow{2}{*}{0.99} & \multirow[t]{2}{*}{$h_{B S}^{2}$} & $* * 41.77$ & 7.12 & a54.74 & $5 \times 3$ & & & \\
\hline & & $* * 15.80$ & $1.17-$ & g44.71 & $6 \times 3$ & & & \\
\hline 0.05 & $h_{N S}^{2}$ & $* * 23.25$ & 3.41 & c50.97 & $5 \times 4$ & 0.93 & $\mathrm{a} 41.36$ & 4 \\
\hline 6.01 & $\bar{a}$ & $* * 11.44$ & 0.26 & $\mathrm{f} 46.09$ & $6 \times 4$ & 1.00 & $\mathrm{~d} 35.90$ & 5 \\
\hline 0.66 & $G_{\tilde{i}}$ & $* * 45.36$ & 7.43 & b53.33 & $6 \times 5$ & $0.73^{-}$ & bc36.69 & 6 \\
\hline 1.46 & $G_{i} \%$ & & 0.21 & S.E & & 0.16 & S.E & \\
\hline
\end{tabular}

وتعد صفة حاصل النبات الفردي محصلة لمكونات الحاصل ومن اكثر الصفات تعقيدا، وتثبر نتائج (الجدول 7) الى ان الاب (5) أحرز أعلى متوسط حسابي وبلغ 23.44، غم وباختلاف معنوي عن الإباء 1 و 3 و6، وحازت الإباء 2 و 5 و 6 على

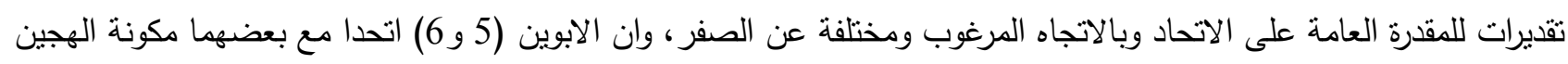

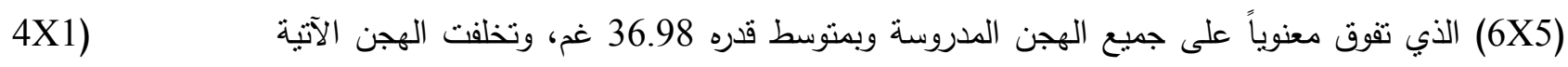

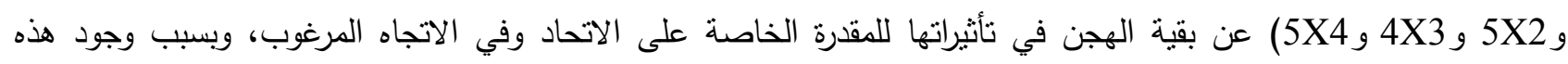

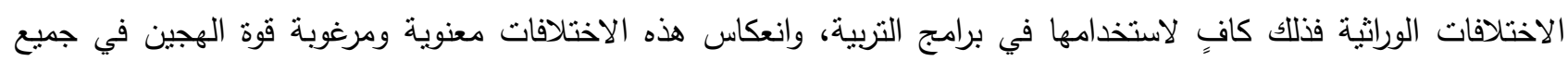
الهجن باستثناء الهجن المذكورة انفاً اذ لم تأتلف للمقدرة الخاصة على الاتحاد، وهذه النتيجة تتوافق مع الصفار والصواف لتهاف (2012). وعند الرجوع للجدول نفسه نلاحظ وجود نباين سيادي أعلى من التباين الإضافي وانخفاض قيمة التوريث بالمعنى الضيق وتجاوز قيمة معدل درجة السيادة اكبر من واحد، وبالتالي يمكن استثمار هذه الهجن المتميزة بقوة هجين عالية وذللك ناجم عن السيادة الفائقة وتحدد انعزالات فائقة الحدود Transgressive Segregation المرغوب فيها، وللاستفادة منها في برامج التربية لاستتباط Yadav et al., (2017) أصناف بمواصفات ذي إنتاجية ونوعية جيدة، واتفقت نتائجنا مع ما توصل إليه الباحثين و Kumar et al., (2017) 


$$
\text { احمد هواس عبد الله أنيس و عبد القادر حميدي جاسم الجبوري }
$$

الجدول 7: المتوسطات للآباء وهجنها وتأثيراتهما وقوة الهجين لصفة حاصل النبات الفردي (غم)

\begin{tabular}{|c|c|c|c|c|c|c|c|c|}
\hline \multicolumn{2}{|c|}{ مكونات التباين المظهري } & \multirow{2}{*}{ | قالمقارنة مين } & \multirow{2}{*}{$\begin{array}{r}\text { تأثير المقدرة } \\
\\
4.00\end{array}$} & \multirow{2}{*}{$\begin{array}{l}\text { المتوسطات } \\
\text { c31.79 } \\
\end{array}$} & \multirow{2}{*}{ الهجن } & \multirow{2}{*}{ تأثير المقدرة } & \multirow{2}{*}{ المتوسطات } & \multirow{2}{*}{$\begin{array}{l}\text { الإباء } \\
1\end{array}$} \\
\hline 0.30 & $\sigma_{E}^{2}$ & & & & & & & \\
\hline 0.20 & S.E & $* * 30.79$ & 2.13 & fg27.62 & $3 \times 1$ & & & \\
\hline 1.85 & $\sigma_{A}^{2}$ & 7.48 & $0.76^{-}$ & h24.16 & $4 \times 1$ & & & \\
\hline 1.46 & S.E & $* * 16.77$ & 0.63 & fg27.38 & $5 \times 1$ & & & \\
\hline 31.59 & $\sigma_{D}^{2}$ & **53.67 & 4.53 & $\operatorname{cd} 30.76$ & $6 \times 1$ & & & \\
\hline 10.94 & S.E & **52.12 & 6.08 & b34.07 & $3 \times 2$ & 1.63 & ab22.39 & 2 \\
\hline & $\sigma_{G}^{2}$ & **29.96 & 1.89 & de29.76 & $4 \times 2$ & & & \\
\hline 33.44 & & $* * 20.32$ & $1.03^{-}$ & efg28.21 & $5 \times 2$ & & & \\
\hline 33.74 & $\sigma_{P}^{2}$ & $* * 49.76$ & 4.81 & b33.54 & $6 \times 2$ & & & \\
\hline & & 7.16 & $1.03^{-}$ & h24.54 & $4 \times 3$ & $0.66^{-}$ & bc21.12 & 3 \\
\hline 090 & $h_{B S}^{2}$ & $* * 22.26$ & 1.71 & ef28.66 & $5 \times 3$ & & & \\
\hline 0.99 & & $* * 26.29$ & 0.23 & g26.67 & $6 \times 3$ & & & \\
\hline 0.05 & $h_{N S}^{2}$ & $* 14.09$ & $0.08^{-}$ & g26.75 & $5 \times 4$ & $0.77-$ & ab22.90 & 4 \\
\hline 5.84 & $\bar{a}$ & $* * 37.32$ & 5.12 & c31.45 & $6 \times 4$ & 0.59 & a23.44 & 5 \\
\hline 0.56 & $G_{i}$ & **53.47 & 8.29 & a35.98 & $6 \times 5$ & 0.08 & $\mathrm{~d} 15.68$ & 6 \\
\hline 2.07 & $G_{i} \%$ & & 0.23 & & S.E & 0.17 & S.E & \\
\hline
\end{tabular}

الجدول 8: البعد الوراثي بين الإباء الداخلة في الاراسة حسب التحليل العنقودي

\begin{tabular}{|c|c|c|c|c|c|c|}
\hline \multirow{2}{*}{ Case } & \multicolumn{7}{|c|}{ Squared Euclidean Distance } \\
\cline { 2 - 7 } & $\mathbf{1}$ & $\mathbf{2}$ & $\mathbf{3}$ & $\mathbf{4}$ & $\mathbf{5}$ & $\mathbf{6}$ \\
\hline 1 & .000 & 34.00 & 116.44 & 265.63 & 127.27 & 799.49 \\
\hline 2 & 34.00 & .000 & 66.67 & 137.39 & 37.79 & 941.07 \\
\hline 3 & 116.44 & 66.67 & .00 & 93.14 & 60.83 & 1190.21 \\
\hline 4 & 265.63 & 137.39 & 93.14 & .00 & 90.69 & 1131.56 \\
\hline 5 & 127.27 & 37.79 & 60.83 & 90.69 & .00 & 1257.24 \\
\hline 6 & 799.49 & 941.07 & 1190.21 & 1131.56 & 1257.24 & .00 \\
\hline
\end{tabular}

\section{الاستنتاجات}

نستتج مما سبق ان الابوين 2 و 5 تميزا بمقدرة عامة على الاتحاد بالاتجاه المرغوب ولصفتي وزن 1000 حبة وحاصل النبات الفردي وحصولنا على هجينين متميزين (6X2) و (6X5) ذي مقدرة اتحاد خاصة مرغوبة ناتجة من اباء مرغوبة وتعطي قوة هجين موجبة ومعنوية قياساً إلى أفضل أب وكذلك وقوع جميع الصفات تحت تأثير الفعل الجيني السيادي، وهذا ما يؤهل هذه الهجن لتكون مادة مهمة للانتخاب خلال الأجيال الانعزالية المتأخرة. 


\section{المصادر العربية}

انيس، احمد هواس عبد اله (2016). علاقة البعد الوراثي في التحليل العنقودي للصفات الكمية لتقديرات بعض المعالم الوراثية

لتهجينات نبادلية في حنطة الخبز (Triticum aestivum L.). المجلة المصرية للعلوم التطبيقية، 30(2)، 130-

انيس، احمد هواس عبداله؛ الزبيدي، خالد محمد داؤد؛ العبيدي، داؤد سلمان مدب؛ العبيدي، صدام إبراهيم يحيى (2017). التحليل الوراثي لحاصل الحبوب وبعض مكوناته في التهجين التبادلي الجزئي لتراكيب وراثية من حنطة الخبز . مجلة حوليات

$$
\text { العلوم الزراعية بمشتهر • مصر • 56(1)، 1-10. }
$$

البدراني، نبيل طه يونس؛ يوسف، نجيب قاقوس؛ الحمداني، غادة عبداله طه (2012). التحليل الوراثي لمتوسطات الأجيال ذاتية

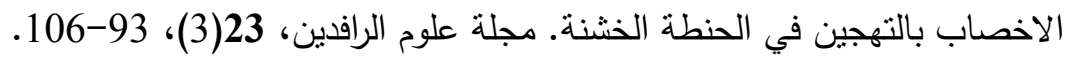

سباهي، جليل (2011). دليل استخدام الأسمدة الكيمياوية والعضوية في العراق. نشرة وزارة الزراعة العراقية. شاهرلي، مخلص؛ خوري، بولص (2012). السلوكية الوراثية لبعض الصفات المرتبطة بالغلة الحبية في القمح القاسي. مجلة جامعة

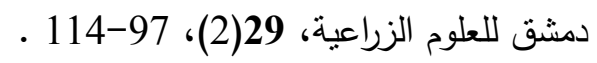

الصفار، رائد سالم احمد؛ الصواف، زهراء خزعل حمدون (2012). تقدير قوة الهجين والتوريث في الحنطة الناعمة Triticum aestivum L.

العذاري، عدنان حسن محمد (1999). أساسيات في الوراثة. وزارة التعليم العالي والبحث العلمي. جامعة الموصل.

العطرات، مهدي؛ صبوح، محمود؛ العك، وليد (2014). التباين الوراثي ودرجة التوريث وقوة الهجين في تحسين الغلة الحبية

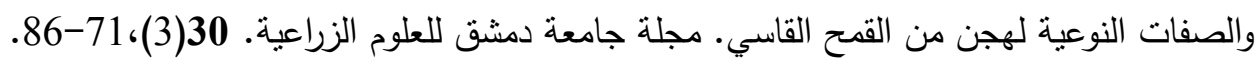

علي، عبده الكامل عبداله (1999). الغزارة الهجينية والفعل الجيني في الذرة الصفراء (Zea mays L) ـ أطروحة دكتوراه، كلية الزراعة والغابات، جامعة الموصل، العراق.

معلا، محمد يحيى؛ حربا، نزار علي (2011). دراسة قوة الهجين والمقدرة العامة والخاصة على الائتلاف للغلة وعناصرها للى

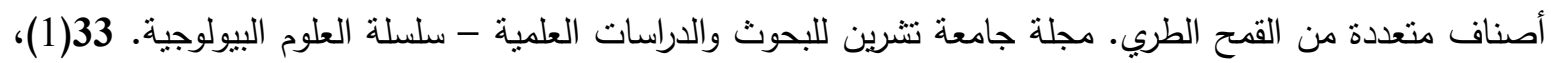
$.24-9$

\section{المصادر الأجنبية}

Agrarwal, V.; Ahmed, Z. (1982). Heritability and genetic advance in triticale. Indian J. Agric. Res., 16, 19-23.

Allard, R. W. (1960). "Principles of Plant Breeding". John Wiley and Sons, Inc, New York, London. $485 \mathrm{p}$.

Al-Zubaidy, K.M.D.; Al-Falahy, M.A.H. (2016). "Principle and Procedures of Statistics and Experimental Design". Duhok University Press, Iraq.

Ferrari, E.D.; Ferreira, V.A.; Grassi, E.M.; Picca, A.M.T.; Antonio, H. (2018 ). Paccapelo genetic parameters estimation in quantitative traits of a cross of triticale ( $x$ Triticosecale W.). Open Agr. 3, 25-31

Griffing, B. (1956). Concept of general and specific combining ability in relation to diallel crossing system. Aust. J. Biol. Sci. 9, 463-493.

Kalhoro, F.A.; Rajpar, A.A.; Kalhoro, S.A.; Mahar, A.; Ali, A.; Otho, S.A.; Soomro, R.N.; Ali, F.; Baloch, Z.A. (2015). Heterosis and combing ability in $\mathrm{f1}$ population of hexaploid wheat (Triticum aestivum L.) . American J. Plant Sci. 6, 1011-1026.

Kempthorne, O. (1957). "An Introduction to Genetic Statistics". John Wiley and Sons, NewYork, U.S.A.

Kempthorne, O.; Curnow, R. N. (1961). The partial diallel cross. Biometrics, 17, 229 - 250. 


$$
\text { احمد هواس عبد الله أنيس و عبد القادر حميدي جاسم الجبوري }
$$

Khodadadi, E.; Aharizad, S.; Sabzi, M.; Shahbazi, H.; Khodadadi, E. (2012). Combining ability analysis of bread quality in wheat. Annals Bio. J. Res. Iran, 3(5), 2464-2468.

Kumar, S.; Singh, S.K.; Singh, L.; Gupta, S.K.; Prashant, V.; Yadav, P.C.; Singh, Y.P.; Kumar, S. (2017). Heterosis and inbreeding depression for grain yield and related morphophysiological characters in wheat (Triticum aestivum L.). Int. J. Curr. Microbiol. App. Sci India .6(10), 1352-1364.

Saeed, M.; Khalil, I.H. (2017). Combining ability and narrow-sense heritability in wheat (Triticum aestivum L.) under rainfed environment. Sarhad J. Agr. Pakistan. 33(1), 22-29.

Thomas, N.; Marker, S.; Lal, G.M.; Dayal, A. (2017). Study of heterosis for grain yield and its components in wheat (Triticum aestivum) over normal and heat stress condition. J. Ph. and Phy. Turkey. 6(4), 824-830.

Unay, A.; Basal, H.; Konak, C. (2004). Inheritance of grain yield in a half - diallel maize population. Turk. J. Agric. 28, $239-244$.

Yadav, J.; Sharma, S.N.; Jakhar, M.L.; Shweta (2017). Combining ability analysis for yield and its components in bread wheat (Triticum aestivum L.) over environments. Int. J. P.Sci. 12(2), 95-101 .

Yadav, H.K.; Singh, S.P. (2011). Inheritance of quantitative traits in opium. Genetika. 43(1), 113 128. 